Journal of Case Reports 2019;9(1):33-35

\title{
Rare Presentation of Acute Intestinal Obstruction: Cecal Volvulus with Malrotation
}

\author{
Pramod $\mathrm{S}^{1}$, Ganesh Kumar ${ }^{2}$ \\ Departments of ${ }^{1}$ Paediatric Surgery and ${ }^{2}$ Surgery, Kempegowda Institute of Medical Sciences and Research Institute, Bangalore, \\ Karnataka, India.
}

\section{Corresponding Author: \\ Dr. Pramod S}

Email: pramodbmc76@gmail.com

This is an Open Access article distributed under the terms of the Creative Commons Attribution License (creativecommons.org/ licenses/by/3.0).

\begin{abstract}
Background: Cecal volvulus occurs due to torsion or hyper-flexion of an enlarged, poorly fixed hyper-mobile cecum. Intestinal malrotation occurs due to incomplete or faulty rotation and fixation of the gut in fetal life. Combination of these two conditions is rare. Case Report: Here we report a 15 year old male child presenting with acute onset pain abdomen with vomiting. On evaluation child was found to have cecal volvulus and associated malrotation. The child was treated successfully with surgery. Conclusion: Cecal volvulus is medical emergency that requires high index of clinical suspicion for its diagnosis.
\end{abstract}

Keywords: Abdomen, Abdominal Pain, Cecal Diseases, Intestinal Volvulus, Rotation.

\section{Introduction}

Cecal volvulus accounts for $30 \%$ of all colonic volvulus [1]. It occurs secondary to torsion or hyper-flexion of an enlarged, poorly fixed hypermobile cecum. Intestinal malrotation occurs due to incomplete or faulty rotation and fixation of the gut during the $5^{\text {th }}$ to $11^{\text {th }}$ week of fetal life. A combination of both is rare. It is associated with significant morbidity and mortality because of the diagnostic delay as a result of rarity of the condition and altered anatomy [2]. Treatment and prognosis depends on the viability of the dilated cecum. Definite treatment of the cecal volvulus is surgery. Here we present a 15 year old male child presenting with cecal volvulus and associated malrotation. The child was treated successfully with surgery.

\section{Case Report}

A 15 year old male child presented with history of pain abdomen since last two days. The pain present in the upper quadrant of the abdomen was intermittent and spasmodic. The intensity of pain had increased since last 12 hours. Pain abdomen was associated with vomiting. Initially vomiting was non-bilious but later bilious. Child had not passed stools since last three days. There was no history of fever, blood in stools, or urinary symptoms. On examination the child appeared dehydrated. His vitals were within normal limits. On abdominal examination fullness was present in the epigastric region [Fig.1]. Palpation revealed a mass in the epigastric region extending upto the umbilicus. Mass was resonant to percuss. Child had diffuse tenderness all over the abdomen. Rectal examination revealed an empty rectum.

Blood investigations were within normal limits. An X-ray of the abdomen showed a large dilated bowel loop in the upper abdomen. A computed tomography of the abdomen revealed a grossly dilated cecum in the epigastrium [Fig.2,3] with sub-acute obstruction. With the above findings the child's parents were counselled about the need of surgery. 
The child underwent laparotomy with a right upper quadrant transverse incision. Intraoperatively cecum was present in the left upper abdomen with volvulus of two and half turns in clockwise direction [Fig.4]. The cecum was de-rotated. The duodenal flexure was to the right of the vertebrae suggestive of malrotation. Adhesions were present between large bowel loops and duodenum. Cecum was healthy and nongangrenous post-derotation [Fig.5]. The adhesive bands between the large bowel loops and duodenum were released. The duodenum was straightened and bowel was placed in non-rotation. Small bowel loops were placed in to the left of abdomen and large bowel loops to the right of abdomen. An appendectomy was done.

Post-operatively child was started on feeds on post-operative day five. The child was discharged on post-operative day eight. Child is on regular follow up since two years and is doing fine.

\section{Discussion}

Cecal volvulus incidence ranges from 2.8 to 7.1 per million population [3]. Cecal volvulus is a rare cause of large bowel obstruction, second only to sigmoid volvulus in its frequency of occurrence of large bowel volvulus. Cecal volvulus presents in two forms. The common presentation form is axial rotation of the cecum and the ileum around the mesentery and the less common form is cecal bascule, where the bowel folds up on itself [4]. The main pathogenesis of cecal volvulus may be attributed to the long right colonic mesentery. Although in $10-15 \%$ of the population retro-peritoneal fixation of the cecum and ascending colon fails, cecal volvulus remains rare [5]. Common causes are usually idiopathic or congenital bands. The precipitating factors includes the conditions that impair the emptying of the colon due to stenotic process, post-operative paralytic ileus, pregnancy with cecal dislocation, abdominal surgery requiring mobilization of the right colon and high residual diet.

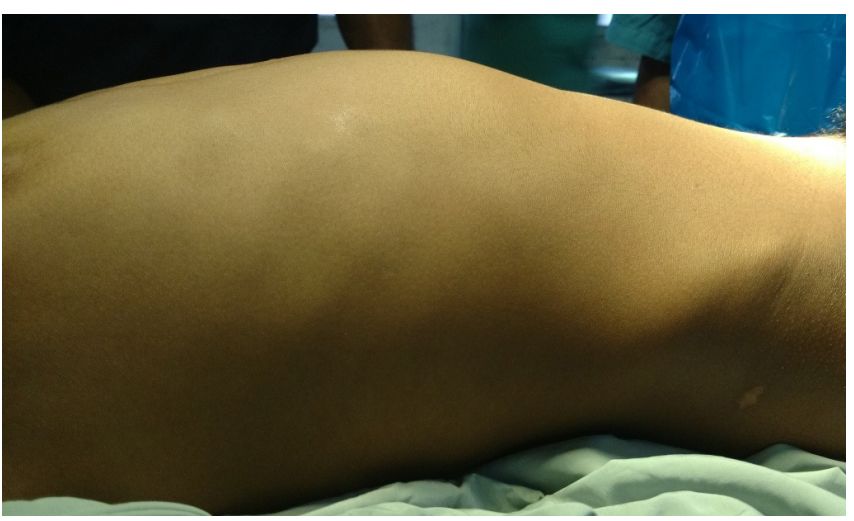

Fig.1: Fullness of epigastium.
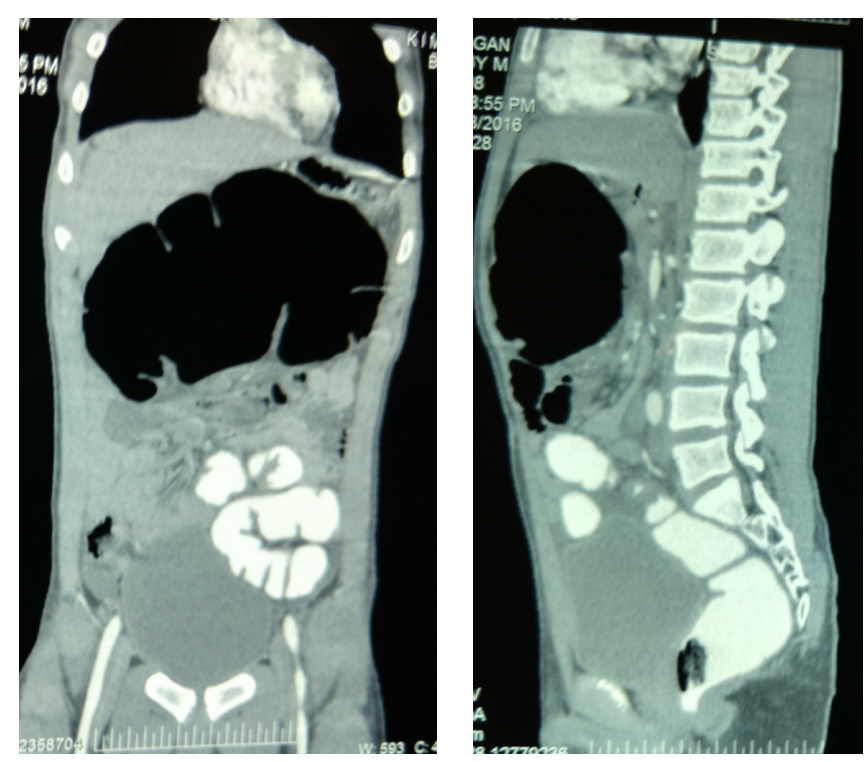

Fig.2,3: CT scan showing grossly dilated cecum.
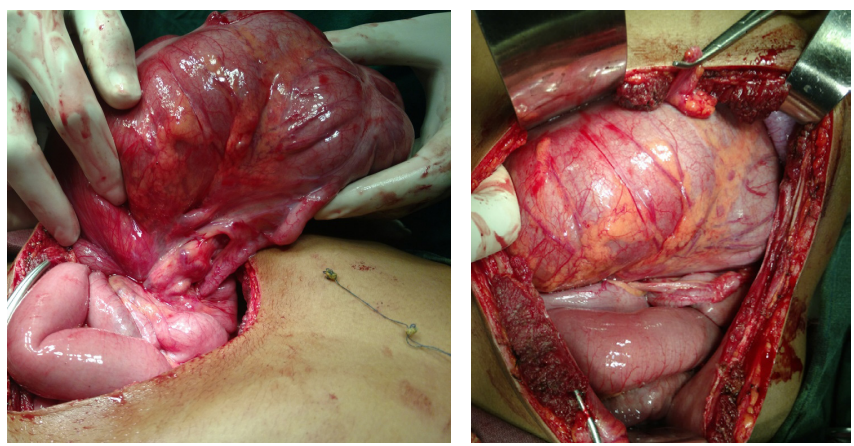

Fig.4,5: Cecal volvulus. 
Cecal volvulus is also one of the modes of presentation of intestinal malrotation. Midgut malrotation has been estimated to occur in approximately 1 in 500 live births [6]. Approximately $85 \%$ of malrotation cases present in the first 2 weeks of life [7]. In adults, intestinal malrotation occurs in $0.2-0.5 \%$ of population $[8,9]$. Since both cecal volvulus and intestinal malrotation are rare, diagnostic delay is common and hence complications occur. The possible etiologies of cecal volvulus in this patient were malrotation and abnormal fixation of bowel.

Initial investigation is erect X-ray abdomen in these patients which show a large dilated bowel loop with air fluid levels. Investigation of choice is CT abdomen. CT features of cecal volvulus are 'whirl' sign, intestinal twisting, and the clockwise or counter-clockwise direction of the volvulus [10]. CT is also helpful in cases of associated malrotation. CT finding in malrotation includes abnormal relationship between the superior mesenteric artery (SMA) and superior mesenteric vein (SMV). The initial investigation in the present child was a X-ray which showed a large dilated bowel loop in the upper abdomen. CT scan was suggestive of a cecal volvulus.

Surgery remains the main definite treatment for cecal volvulus. The present child underwent a derotation of cecal volvulus segment followed by Ladd's procedure. The various options include manual detorsion alone, untwisting with cecopexy, cecostomy and resection with anastomosis. In case of perforation or gangrene of the volvulus segment, a resection and anastomosis or a colostomy may be required. The best long-term results have been with resection and primary anastomosis. Isolated reports of laparoscopic cecopexy in emergency setting have been described more recently [11].

\section{Conclusion}

Cecal volvulus with malrotation is rare condition. It is important to diagnose this condition at the earliest to prevent complication. Surgery is the treatment of choice.

Contributors: PS: manuscript writing, patient management; GK: manuscript editing, patient management. PS will act as guarantor. Both authors approved the final version of this manuscript.

Funding: None; Competing interests: None stated.

\section{References}

1. Habre J, Sautot-Vial N, Marcotte C, Benchimol D. Caecal volvulus. Am J Surg. 2008;196:e48-49.

2. Rogers RL, Harford FJ. Mobile cecum syndrome. Dis Colon Rectum. 1984;27:399-402.

3. Rabinovici R, Simansky DA, Kaplan O. Cecal volvulus. Dis Colon Rectum. 1990;33:765-769.

4. Ballantyne GH, Brandner MD, Beart RW, Llstrup DM. Volvulus of the colon. Incidence and mortality. Ann Surg. 1985;202:83-92.

5. Samuel M, Boddy SA, Nicholls E. Large bowel volvulus in childhood. Aust NZ J Surg. 2000;70:258-262.

6. Torres AM, Ziegler MM. Malrotation of the intestine. World J Surg. 1993;17:326-331.

7. McIntosh R, Donovan EJ. Disturbances of rotation of the intestinal tract. Am J Dis Child. 1939;56:116-166.

8. Frantzides CT, Cziperle DJ, Soergel K, Stewart E. Laparoscopic Ladd procedure and cecopexy in the treatment of malrotation beyond the neonatal period. Laparosc Endosc Percutan Tech. 1996;6:73-75.

9. Mazziotti MV, Strasberg SM, Langer JC. Intestinal rotation abnormalities without volvulus: the role of laparoscopy. J Am Coll Surg. 1997;185:172-176.

10. Delabrousse E, Sarlieve P, Sailley N. Cecal volvulus: CT findings and correlation with pathophysiology. Emerg Radiol. 2007;14:411-415.

11. Madiba TE, Thomson SR. The management of cecal volvulus. Dis Colon Rectum. 2002;45:264-267. 